\title{
Proliferating cell nuclear antigen expression in papillary thyroid carcinoma
}

\author{
H Ando, $\mathrm{H}$ Funahashi, M Ito, T Imai, H Takagi
}

\begin{abstract}
Aims-To correlate the expression of proliferating cell nuclear antigen (PCNA) with various clinicopathological features of papillary thyroid carcinoma.

Methods-Sections from 131 formalin fixed, paraffin wax embedded papillary thyroid carcinomas were stained with a monoclonal antibody (PC10) directed against PCNA using the avidin-biotin immunoperoxidase (ABC) method. PCNA immunoreactivity was based on the PCNA labelling index (LI) following evaluation of at least 1000 tumour cells, and expressed as follows: grade A (LI < 10\%), grade $B(10 \% \leqslant L I<25 \%)$, and grade $C$ (LI $\geqslant 25 \%)$. The relation between PCNA expression in these three groups and other clinicopathological factors, such as sex, age, tumour size, nodal metastases, and histological differentiation, were examined.
\end{abstract}

Results-Based on the labelling index, 57 (43.5\%) cases were graded as A, 46 (35.1\%) as $B$, and $28(21.4 \%)$ as $C$. The female:male ratios were 6.13:1 for group $A, 2.83: 1$ for group B, and 2.11:1 for group $C$. The mean (SD) ages of the patients were 39.0 (16.1) in group A, $53.5(14.4)$ in group B, and 55.8 (13.3) years in group $C$. The correlation between age and PCNA grade was strongest in women.

Conclusions-PCNA immunoreactivity is correlated with sex and age in patients with papillary thyroid tumours.

(F Clin Pathol 1996;49:657-659)

Keywords: PCNA, papillary thyroid carcinoma, immunohistochemistry.

Department of

Surgery II, Nagoya

University School of

Medicine, Nagoya,

Japan

H Ando

H Funahashi

T Imai

H Takagi

Department of

Pathology

M Ito

Correspondence to: Dr Masafumi Ito,

Department of Pathology,

Nagoya University Hospital,

65 Tsurumai-cho, Showa-ku,

Nagoya 466, Japan.

Accepted for publication

16 April 1996
The prognosis of patients with papillary thyroid carcinoma is generally good, even if surgical resection is not performed early in the clinical course. However, there is a subset of patients. with a poorer prognosis, in whom the tumour infiltrates the surrounding cervical tissues which cannot be cured even with radical treatment. These patients do not always present with histologically poorly differentiated or anaplastic carcinomas.

Recently, there have been several studies examining the proliferative activity of various malignant tumours using immunohistochemical methods. Few such studies have examined the proliferative activity of papillary thyroid carcinomas. These studies suggest that there is a relation between general clinical course and proliferative activity in papillary thyroid can- cer. ${ }^{12}$ However, there are patients who follow a clinically malignant course although they may have favourable histology. Therefore, we studied proliferating cell nuclear antigen (PCNA) expression in papillary thyroid carcinoma during various clinical stages and related this expression to the other clinicopathological features.

\section{Methods}

Patients ( $n=131$ ) surgically treated for papillary thyroid carcinoma at the Department of Surgery II, Nagoya University Hospital between 1981 and 1993 were studied. Papillary carcinoma was confirmed histologically in all 131 patients. Tumours that underwent anaplastic transformation and were associated with follicular carcinomas were excluded. The mean (SD) age of the patients overall was 47.7 (16.7) years (range 7-83 years) and of the 102 female and 29 male patients were 45.2 (15.8) and 56.7 (16.8) years, respectively. Surgical treatment comprised total thyroidectomy (123 patients), subtotal thyroidectomy (one patient), lobectomy (four patients), and tumour excision (four patients).

\section{IMMUNOSTAINING}

Specimens were fixed routinely in $10 \%$ formalin and embedded in paraffin wax. Sections, $3 \mu \mathrm{m}$ thick, were mounted on poly-L-lysine coated glass slides and deparaffinised using xylene and graded alcohol. The sections were then washed with water and incubated with normal goat serum for 10 minutes to reduce non-specific antibody binding. The primary monoclonal mouse anti-PCNA antibody (NCL-PCNA, PC10; Novocastra Laboratories Ltd, UK) was diluted 1 in 200 and applied to the sections overnight at $4^{\circ} \mathrm{C}$. Sections were rinsed in phosphate buffered saline (PBS) and then immersed in $0.03 \% \mathrm{H}_{2} \mathrm{O}_{2}$ in methanol for 20 minutes to block endogenous peroxidase activity. The secondary antibody, a biotinylated horse anti-mouse immunoglobulin, was applied for 40 minutes, sections were rinsed in PBS, and an avidin-biotin peroxidase complex reagent (Vectastain ABC kit, Vector Laboratories, Burlingame, California, USA) applied for 30 minutes at room temperature. Diaminobenzidine (Sigma, St Louis, Missouri, USA) was used as the chromogen. Sections were lightly counterstained with Mayer's haematoxylin.

Quantitative analysis of PCNA expression was conducted by determining the ratio of positively to negatively stained cells (labelling index, LI). At least five representative high power fields $(\times 400)$ were examined by light 
Table 1 Clinicopathological features according to PCNA grade. Results are expressed as mean (SD) unless stated othewise

\begin{tabular}{|c|c|c|c|c|c|c|c|c|c|c|}
\hline \multirow{2}{*}{$\begin{array}{l}\text { PCNA } \\
\text { grade }\end{array}$} & \multirow{2}{*}{\multicolumn{2}{|c|}{$\begin{array}{l}\text { Female:male ratio } \\
\text { (female/male) }\end{array}$}} & \multirow{2}{*}{\multicolumn{2}{|c|}{ Mean age (years) }} & \multicolumn{4}{|c|}{ Mean age by sex } & \multirow[b]{2}{*}{ Tumour size $(\mathrm{cm})$} & \multirow{2}{*}{$\begin{array}{l}\text { Cases of poorly } \\
\text { differentiated } \\
\text { carcinoma (\%) }\end{array}$} \\
\hline & & & & & Male & & Female & & & \\
\hline A & 6.13: $1(49 / 8)$ & $\star$ & $39.0(16.1)$ & \}$\left.^{\star}\right|^{\star}$ & $50.3(24.1)$ & \}$^{\star}$ & $37.2(4.0)$ & \}$\left.^{\star}\right\}^{\star}$ & $3.3(4.0)$ & $10 / 57(17.5)$ \\
\hline B & 2.83: $1(34 / 12)$ & & $53.5(14.4)$ & & $61.8(11.8)$ & \} & $50.5(14.2)$ & & $3.4(2.5)$ & $13 / 46(28.3)$ \\
\hline $\mathrm{C}$ & $2.11: 1(19 / 9)$ & & $55.8(13.3)$ & & $55.6(14.2)$ & & $56.2(12.8)$ & & $3.7(2.8)$ & $6 / 28(21.4)$ \\
\hline
\end{tabular}

$\mathrm{A}=$ Labelling index $(\mathrm{LI})<10 \% ; \mathrm{B}=10 \% \leqslant \mathrm{LI}<25 \% ; \mathrm{C}=\mathrm{LI} \geqslant 25 \%$.

microscopy. More than 1000 tumour cell nuclei were counted in each specimen, and the percentage of PCNA positive cells was calculated. Tumours were assigned to one of three groups based on the LI (group A, LI $<10 \%$; group $\mathrm{B}, 10 \% \leqslant \mathrm{LI}<25 \%$; group $\mathrm{C}$, LI $\geqslant 25 \%)$. Some of the tumours in each group were also immunostained with the MIB-1 monoclonal antibody (MBL, Nagoya, Japan) for comparison. MIB-1 immunostaining was performed as for PCNA immunostaining except that the specimens were pretreated in a microwave oven and the antibody was diluted 1 in 50. Statistical analysis was performed using Fisher's exact probability test and Student's $t$ test; $\mathrm{p}<0.05$ was considered significant.

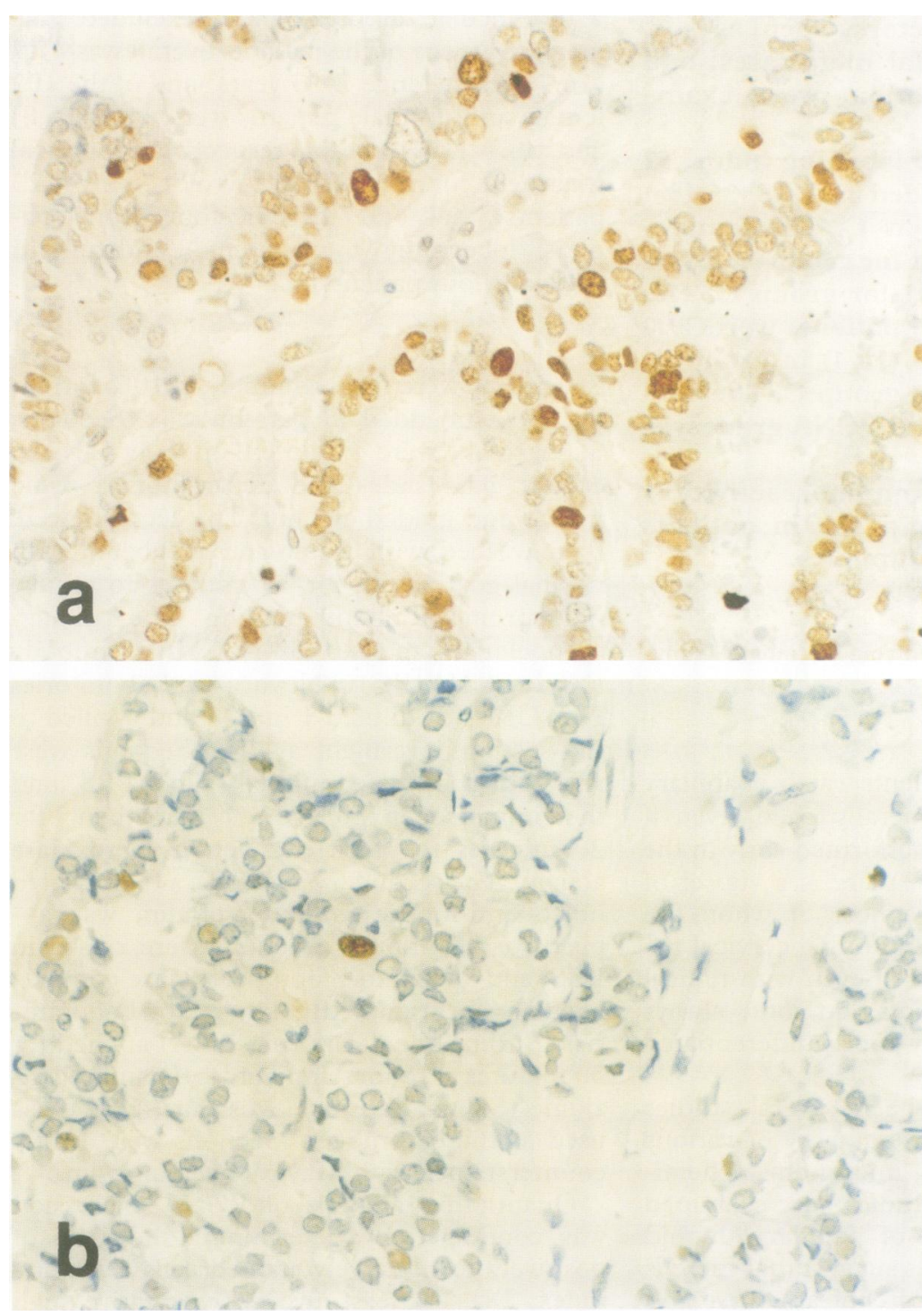

Figure 1 (a) Strong (grade C) and (b) weak (grade A) PCNA immunostaining.

\section{Results}

Based on the labelling index, 57 (43.5\%) tumours were assigned to group A, 46 (35.1\%) to group $B$, and $28(21.4 \%)$ to group C (fig 1 ). Figure 2 shows the distribution of all 131 patients according to both PCNA grade and sex. The female patients were divided into two groups based on age ( $0-49$ years, 50 years and older). The ratios of female:male patients in 8 each group were: 6.13:1 in group $A, 2.83: 1$ in $\overrightarrow{0}$ group $B$ and $2.11: 1$ in group $C$. The percentage of female patients aged 0-49 years of was $70.2 \%$ in group $\mathrm{A}, 30.4 \%$ in group $\mathrm{B}$ and $17.9 \%$ in group $\mathrm{C}$. The mean (SD) age of patients in each group was 39.0 (16.1) in group A, 53.5 (14.4) in group $B$, and 55.8 (13.3) years in group C. When female patients were considered alone, there was a good correlation between the mean age at diagnosis and the PCNA grade. This was not the case for men (table 1).

There was no significant difference in the average maximal diameters of the tumours assigned to the three groups (table 1). There was no significant correlation between PCNA grade and the presence or absence of lymph node metastases (table 2). Twenty nine $(22.1 \%)$ tumours were histologically poorly differentiated papillary carcinomas with either a solid or trabecular, or both, pattern. PCNA 옹 reactivity by group for these poorly differentiated carcinomas was $17.5 \%(10 / 57)$ in group A, $28.3 \%$ (13/46) in group B and $21.4 \%$ (six of 28 ) in group C. PCNA immunoreactivity was uniform in tumours with papillary and solid/ trabecular patterns (table 1 ).

Disease recurred in $23(17.6 \%)$ patients (12 men and 11 women) following surgery; more $\widetilde{N}$

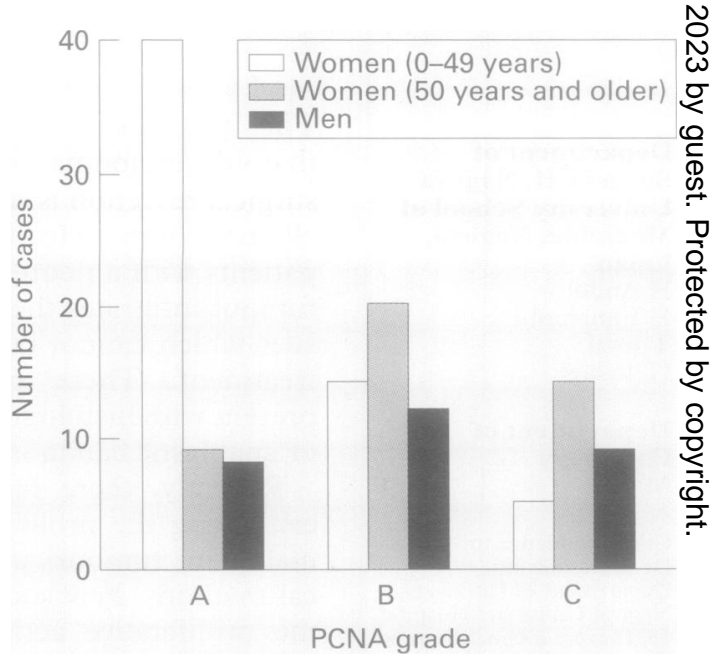

Figure 2 Distribution of patients according to sex and PCNA grade. Women are divided into two groups based on age (0-49 years, 50 years and older). 
Table 2 Relation between lymph node status and PCNA grade. Results expressed as $n(\%)$

\begin{tabular}{llrl}
\hline & \multicolumn{2}{l}{$P$ PCNA grade } \\
\cline { 2 - 4 } $\begin{array}{l}\text { Lymph node } \\
\text { status }\end{array}$ & \multicolumn{1}{l}{$A$} & \multicolumn{1}{l}{$B$} & \multicolumn{1}{c}{$C$} \\
\hline pN0 & $6(11.5)$ & $8(26.7)$ & $2(9.5)$ \\
pN1 & $46(88.5)$ & $30(73.3)$ & $21(90.5)$ \\
Total & 52 & 38 & 23 \\
\hline
\end{tabular}

pN0 = regional lymph node metastases absent.

pN1 = regional lymph node metastases present.

men $(41.4 \%)$ than women $(10.8 \%)$ relapsed. The recurrence ratios according to PCNA grade were as follows: $10.5 \%$ (six of 57 ) in group A, 28.3\% (13/46) in group B and $14.3 \%$ (four of 28 ) in group C. The prognosis for patients in group A was the most favourable of the three groups, although there was no correlation between PCNA expression and overall prognosis.

Ten cases were selected at random from each group and immunostained with MIB-1; however, MIB-1 positivity was rarely observed regardless of the PCNA grade.

\section{Discussion}

PCNA is a factor for DNA polymerase delta, is synthesised during the late $\mathrm{Gl}$ to $\mathrm{S}$ phase of the cell cycle and has been confirmed to be cyclin. ${ }^{3}$ Recently, many investigators have applied PCNA immunostaining to clinical specimens in order to estimate the malignant nature or prognosis of various neoplasms. In the current study, we found that some cases of papillary thyroid carcinoma stained strongly for PCNA. Previous studies have suggested that the proliferative activity of papillary carcinomas in terms of the PCNA positive ratio is generally less than $10 \% .^{2}$ However, we found several cases with PCNA immunostaining labelling indexes of more than $50 \%$.

One of our major findings was that PCNA expression in papillary thyroid carcinoma correlates with the age and sex of the patients. The female:male ratio was lower, and the mean age of patients was higher in tumours strongly expressing PCNA than those with weak expression. The correlation between age and PCNA expression was especially strong for women. This result is interesting because the most important prognostic factors in papillary carcinoma are the age and sex of the patient, and therefore PCNA grade cannot be used as an independent prognostic factor. Other clinicopathological features such as a tumour size, lymph node metastases and the degree of differentiation ${ }^{5}$ did not correlate with PCNA expression.

It has been suggested that PCNA activity is not necessarily associated with DNA synthesis, but may be involved in unscheduled DNA repair throughout the cell cycle. ${ }^{67}$ The proportion of PCNA positive cells found in many tumours exceeds that expected for the proportion of proliferating cells as assessed by other methods. ${ }^{8}$ We also found that some papillary carcinomas overexpressed PCNA and that MIB-1 immunostaining indicated the real proliferative activity of the tumour. ${ }^{9}$ Some authors have suggested that PCNA overexpression is a result of cytokine action. ${ }^{10}{ }^{11}$ In the current study, there was no correlation between PCNA expression and lymphocytic infiltration. In conclusion, as yet unknown factors regulated by the age and sex of patients with papillary thyroid carcinoma may be related to PCNA expression.

1 Yoshida A, Kamma H, Asaga T, Masuzawa C, Kawahara S, Miura T, et al. Proliferative activity in thyroid tumors. Cancer 1992;69:2548-52.

2 Shimizu T, Usuda N, Yamada T, Sugenoya A, Iida F. Proliferative activity of human thyroid tumors evaluated by proliferating cell nuclear antigen/cyclin immunohistochemical studies. Cancer 1993;71:2807-12.

3 Bravo R, Frank R, Blundell PA, MacDonald-Bravo $H$. Cyclin/PCNA is the auxiliary protein of DNA polymerasedelta. Nature 1987;326:515-17.

4 Prelich G, Tan CK, Kostura M, Mathews MB, So AG, Downey KM, et al. Functional identity of proliferating cell nuclear antigen and a DNA polymerase-delta auxiliary protein. Nature 1987;326:517-20.

5 Sakamoto A, Kasai N, Sugano H. Poorly differentiated carcinoma of the thyroid: A clinicopathologic entity for a high-risk group of papillary and follicular carcinomas. Cancer 1983;52:1849-55.

6 Toschi L, Bravo R. Changes in cyclin: proliferating cell nuclear antigen distribution during DNA repair synthesis. f Cell Biol 1988;107:1623-28.

7 Shivji KK, Kenny MK, Wood RD. Proliferating cell nuclear antigen (PCNA) is required for DNA excision repair. Cell 1992;69:1-20.

8 Hall PA, Levison DA, Woods AL, Yu CCW, Kellock DB, Watkins JA, et al. Proliferating cell nuclear antigen (PCNA) immunolocalization in paraffin sections: an index of cell proliferation with evidence of deregulated expression in some neoplasms. F Pathol 1990;162:285-94.

9 Rose DSC, Maddox PH, Brown DC. Which proliferation markers for routine immunohistology? A comparison of markers for routine immunohistology? A com
five antibodies. $\mathcal{f}$ Clin Pathol 1994;47:1010-14.

10 Hall PA, Hart I, Goodlad R, Cortes PJ, Lane DP. Expression of proliferating cell nuclear antigen in non-cycling cells. $\mathcal{F}$ Pathol 1992;168:97A.

11 Monaghan P, Perusinghe NP, Nicholson RI, McClelland RA, O'Hare MJ, Lane DP. Growth factor stimulation of proliferating cell nuclear antigen (PCNA) in human breast epithelium in organ culture. Cell Biol Int Rep 1991; 15:561-70. 